Acta Crystallographica Section C

Crystal Structure Communications

ISSN 0108-2701

\section{George Ferguson}

School of Chemistry, The University of St Andrews, St Andrews, Fife KY16 9ST, Scotland

\section{Further developments for Acta Crystallographica Section C}

While Acta Crystallographica Section E: Structure Reports Online, recently became an Open Access journal and is an excellent medium for the dissemination of brief crystal structure reports, Acta Crystallographica Section C: Crystal Structure Communications continues the tradition of a journal supported by reader subscriptions. Section $C$ is a first-rate journal for the rapid publication of full papers describing one or more interesting or unusual crystal structures in significant detail. We invite high-quality detailed studies of novel and challenging crystal and molecular structures of interest in the fields of chemistry, biochemistry, mineralogy, pharmacology, physics and materials science. The unique checking, editing and publishing facilities of the journal ensure the highest standards of structural reliability and presentation, while providing for reports on studies involving special techniques or crystalline materials presenting special difficulties.

Some changes have been made to the 2008 Section C Notes for Authors (see below) to help authors more easily understand the requirements of the journal and thereby prepare their submissions in a style that is fully acceptable for Section $C$.

To assist Co-editors with the initial review of submissions it is now a requirement that the submitting author provide in the _publ_contact_letter section of the CIF a brief statement of what is novel or interesting about the structure(s) that merits publication in Section C. If the Coeditor decides during the review process that publication in Section $C$ is not appropriate for any reason, the authors may be advised to consider a resubmission to Section E or elsewhere. Submissions which report the determination of a structure (i.e. establish connectivity) but have little in the way of significant discussion will not be accepted for publication in Section C. Details of what should be in the Abstract are given in Notes for Authors $\$ 2.3$. Note that the Abstract should clearly state the scientific impact of the study and should not have details of the source or synthesis of the compound(s) studied; these details will be available in the Experimental text. To make clearer what is wanted in the Comment section, there have been significant changes to Notes for Authors $\$ 2.4(d)$ and $\$ 2.4(e)$. The Comment should include:

(d) Novel or unusual aspects of the coordination, geometry, conformation, crystal packing, hydrogen bonding, etc. A discussion of geometry values that agree with established values is not warranted. The Comment should not be just a list of observations about the structure (for example, just stating, without description, that hydrogen-bonding or other weak interactions exist), but should include an analysis and discussion of how these observations help the understanding of the chemical, physical or structural properties of the compound and further the aims of the study stated in the opening paragraph, plus a detailed comparison with any closely related structures, where such exist. Numerical values reported in Tables should generally not be repeated in the text.

(e) A discussion of intermolecular interactions, if warranted, should describe the motifs and networks generated, but over-analysing weak interactions or claiming that such interactions are significantly 'structure stabilizing' should be avoided.

Dr C. Glidewell has resigned from being Deputy Editor but will continue as a valued Coeditor; it is a great pleasure to thank him warmly for his help and wise advice over the last eight years. I am also grateful for the assistance of Dr A. Linden and that of the Chester Editorial staff in the review of the Section C Notes for Authors. Dr. Linden will be taking over from me as Section Editor of Section $C$ in August 2008 and following Dr Glidewell's suggestion has been appointed Deputy Editor until then. I must also acknowledge and warmly thank P. Robinson (Southern Illinois University, USA) and O. Q. Munro (University of KwaZulu-Natal, South Africa), who have now retired from the Section $C$ editorial board, for their services to the journal and the crystallographic community. I would like to take this opportunity to thank all the Section C Co-editors and the Chester Editorial Office staff for their outstanding contributions to Section $C$ and for the assistance they have given me since I was appointed Section Editor in 1999. 